\title{
Associations Between Genetically Predicted Plasma N-Glycans and Prostate Cancer Risk: Analysis of Over 140,000 European Descendants
}

\author{
Duo Liu ${ }^{1,2, *}$ \\ Jingjing $\mathrm{Zhu}^{2, *}$ \\ Tianying Zhao $\mathbb{D}^{2,3, *}$ \\ Sodbo Sharapov ${ }^{4}$ \\ Evgeny Tiys (D) 4 \\ Lang $\mathrm{Wu}^{2}$
}

'Department of Pharmacy, Harbin Medical University Cancer Hospital, Harbin, People's Republic of China;

${ }^{2}$ Cancer Epidemiology Division,

Population Sciences in the Pacific Program, University of Hawaii Cancer Center, University of Hawaii at Manoa, Honolulu, HI, USA; ${ }^{3}$ Molecular Biosciences and Bioengineering, University of Hawaii at Manoa, Honolulu, HI, USA; ${ }^{4}$ Laboratory of Glycogenomics, Institute of Cytology and Genetics, Novosibirsk, Russia

*These authors contributed equally to this work
Correspondence: Lang Wu

Cancer Epidemiology Division, Population Sciences in the Pacific Program, University of Hawaii Cancer Center, University of Hawaii at Manoa, Honolulu, HI, USA Tel +l 808 564-5965

Email Iwu@cc.hawaii.edu
Background: Previous studies suggest a potential link between glycosylation and prostate cancer. To better characterize the relationship between the two, we performed a study to comprehensively evaluate the associations between genetically predicted blood plasma $\mathrm{N}$-glycan levels and prostate cancer risk.

Methods: Using genetic variants associated with N-glycan levels as instruments, we evaluated the associations between levels of 138 plasma $\mathrm{N}$-glycans and prostate cancer risk. We analyzed data of 79,194 cases and 61,112 controls of European ancestry included in the consortia of BPC3, CAPS, CRUK, PEGASUS, and PRACTICAL.

Results: We identified three $\mathrm{N}$-glycans with genetically predicted levels in plasma to be associated with prostate cancer risk after Bonferroni correction. The estimated odds ratios (95\% confidence intervals) were 1.29 (1.20-1.40), $0.80(0.74-0.88)$, and $0.79(0.72-0.87)$ for PGP18, PGP33, and PGP109, respectively, per every one standard deviation increase in genetically predicted levels of N-glycan. However, the instruments for these N-glycans only involved one to two variants. The proportions of variations that can be explained by the instruments range from $1.58 \%$ to $2.95 \%$ for these three N-glycans.

Conclusion: We observed associations between genetically predicted levels of three N-glycans PGP18, PGP33, and PGP109 and prostate cancer risk. Given the correlated nature of the N-glycans and that many N-glycans share genetic loci, pleiotropy is a major concern. Future work is warranted to better characterize the relationship between N-glycans and prostate cancer.

Keywords: plasma N-glycans, prostate cancer, risk

\section{Introduction}

Prostate cancer is the second most commonly diagnosed cancer and the fifth leading cause of malignancy-related death among males worldwide. ${ }^{1}$ It is projected that, in the United States, 191,930 new prostate cancer cases and 33,330 prostate cancerrelated deaths will occur in $2020 .^{2}$ The survival rate tends to be much worse when cancer is diagnosed at a metastatic stage compared with localized stage. ${ }^{3}$ The Prostate-specific antigen (PSA) blood test has been clinically used for prostate cancer screening. ${ }^{4}$ However, using PSA screening is controversial due to the lack of a clear cutoff point for high sensitivity and specificity, ${ }^{5-7}$ and unclear benefit in reducing mortality in specific populations. ${ }^{8-10}$ Therefore, there are critical needs for better understanding the etiology and identifying effective biomarkers to improve the risk assessment of prostate cancer. 
A large proportion of proteins are modified by covalent addition of glycans. Glycosylation is a post-translational modification that is very important for normal physiological processes. ${ }^{11}$ Multiple studies support potential important roles of glycans in cancer progression. ${ }^{12}$ Alterations in glycosylation are shown to potentially change specific properties related to cancer, such as tumor growth, invasion and metastasis. ${ }^{13-18}$ Different types of glycan alterations have been reported to be related to prostate cancer, such as changes to PSA glycosylation, sialylation and core fucosylation, O-GlcNacylation, cryptic and branched $\mathrm{N}$-glycans, as well as galectins and proteoglycans. ${ }^{19}$ As a major class of protein glycosylation besides O-glycans, $\mathrm{N}$-glycans are known to influence many properties of glycoproteins, such as conformation, solubility, antigenicity, as well as recognition by glycan-binding proteins. ${ }^{20}$ Basic research has suggested potential link between $\mathrm{N}$-glycans and prostate tumorigenesis. For example, $\beta$ 1,6-GlcNAc tri-branched and tetra-branched N-glycans were reported to be linked to prostate cancer metastasis in xenograft models. ${ }^{21}$ In humans, changes in plasma and immunoglobulin $\mathrm{G}$ ( $\mathrm{IgG}$ ) N-glycosylation have been reported to be associated with colorectal cancer in a casecontrol study. ${ }^{22}$ However, limited research has been performed to study the relationship between $\mathrm{N}$-glycans and prostate cancer risk. Direct measurement of glycan levels in a large number of subjects is costly. Furthermore, conventional epidemiological studies could be influenced by several common limitations, such as selection bias, potential confounding, and reverse causation. Herein, to better characterize the relationship between plasma proteome $\mathrm{N}$-glycosylation and prostate cancer, we used an approach of genetic instruments that mimic the design of randomized control trials (RCT). ${ }^{23-25}$

\section{Materials and Methods}

\section{Genetic Instruments for N-Glycan Levels in Plasma}

To determine genetic instruments for plasma levels of $\mathrm{N}$-glycans, we conducted a systematic literature search up to April 30, 2020. We focused on genome-wide association studies (GWAS) for $\mathrm{N}$-glycans attached to all plasma proteins or a single protein that is extensively studied, IgG. After careful assessment, we included two largest and most comprehensive studies, one for $\mathrm{N}$-glycans from all proteins, and the other for IgG glycans. ${ }^{26,27}$ For the assessed $\mathrm{N}$-glycans, their quantification is relative in that a glycan peak was defined as a proportion of glycans that fall into this specific (chromatographic) peak among all glycans. We determined the variant(s) associated with each glycan reported in these studies, and only kept singlenucleotide polymorphisms (SNPs) that were independent from each other for each glycan as genetic instruments. The N-glycome (for example, total plasma or IgG) is a result of the complex interplay between biochemical pathways of N-glycan biosynthesis. ${ }^{28}$ The abundances of glycan species are determined by a partially overlapping set of $\mathrm{N}$-glycan biosynthesis enzymes and availability of common substrates, leading to strong correlations between levels of many $\mathrm{N}$-glycans. Therefore, if a locus harbors a particular gene, a product of which regulates the work of the $\mathrm{N}$-glycan biosynthesis enzyme, this locus will likely show an effect on a wide spectrum of related glycan traits. This leads to the fact that one SNP can be a genetic instrument for a single or several glycan traits.

We selected genetic instruments for total plasma $\mathrm{N}$-glycome traits from the results of a GWAS conducted by Sharapov et al, in 2763 TwinsUK samples. ${ }^{26}$ Briefly, genotyping was conducted with combination Illumina SNP arrays (HumanHap300, HumanHap610Q, 1 M-Duo and 1.2MDuo $1 \mathrm{M}$ ). Standard quality control was applied with SNPs being filtered by sample call rate, minor allele frequency, SNP call rate as well as Hardy Weinberg Equilibrium. Variants were further imputed using IMPUTE2 software with $1000 \mathrm{G}$ Phase 1 version 3 as reference data. Plasma N-glycome quantification of samples were measured by ultra-performance liquid chromatography. ${ }^{29}$ Normalization and batch correction were further performed on harmonized UPLC glycan data. Genome-wide Efficient Mixed Model Association algorithm (GEMMA) was further conducted to assess the kinship matrix and to run linear mixed model regression on variants assuming additive genetic effects. In this study, 14 loci were reported to be associated with levels of 68 different traits at the genome-wide significant level of $P$-value $<5 \times 10^{-8} /(29+1)=1.66 \times 10^{-9}$, where 29 is an effective number of tests (traits) that was estimated. Three other cohorts (QMDiab, SOCCS and PainOR) were further used to replicate the identified associations. We extracted genome-wide summary statistics for 113 studied total plasma N-glycome traits and more than 8 million SNPs from the Zenodo repository [https://zenodo.org/record/ 1298406\#.X9-eQthKiU1]. We selected the genetic instruments for each of 113 glycan traits separately. For each of the traits, we selected all SNPs that showed genome-wide 
significant associations at the level of $P$-value $<1.66 \times$ $10^{-9}$. (as reported in ref26). Selected SNPs were clumped by retaining only one SNP within a $250 \mathrm{~KB}$ window. When there are more than one SNP located at the same chromosome for each glycan of interest, the correlations between these SNPs were estimated using the Pairwise LD function of SNiPA (http://snipa.helmholtz-muenchen.de/snipa/ index.php?task=pairwise ld $)$. Only independent SNPs $\left(\mathrm{R}^{2}\right.$ $<0.1$ based on 1000 Genomes Project Phase 3 version 5 data for European descendants) were retained to create an instrument for each glycan. In total, we selected genetic instruments for 68 total plasma $\mathrm{N}$-glycome traits. For most of the plasma N-glycan traits only one instrumental variable was available, therefore limiting the ability to distinguish between causal relationship and horizontal pleiotropy.

We selected genetic instruments for IgG N-glycome traits from the published results of the GWAS of $77 \mathrm{IgG}$ $\mathrm{N}$-glycome traits conducted by Klaric et al. In this study, four cohorts including CROATIA-Korcula, CROATIAVis, ORCADES (Orkney Complex Disease Study), and TwinsUK were leveraged, with a combined sample size of $8090 .^{25}$ The HapMap2 (release 22) imputed genetic data were analyzed for their associations with 77 UPLC IgG N-glycan traits. $P \leq 2.4 \times 10^{-9}$ was used to determine genome-wide significant associations (Bonferroni corrected for 21 independent glycan traits). ${ }^{30}$ In addition, replication analysis was performed by analyzing data of EGCUT, FINRISK, COGS, and SDRNT1BIO ( $\mathrm{N}=2388$ ). For 19 of 27 significant loci identified, the SNP-glycan associations were replicated at $P \leq 1.9 \times 10^{-3}(0.05 / 27)$. Based on this study, we selected genetic instruments for $70 \mathrm{IgG} \mathrm{N}$-glycome traits.

\section{Association Analysis Between Genetically Predicted N-Glycan Levels and Prostate Cancer Risk}

The prostate cancer GWAS summary statistics data were generated from 79,194 prostate cancer cases and 61,112 controls of European ancestry included in the consortia of BPC3, CAPS, CRUK, PEGASUS, and PRACTICAL. ${ }^{31-33}$ Using the OncoArray platform, 46,939 prostate cancer cases and 27,910 controls were genotyped comprising 570,000 SNPs (http://epi.grants.cancer.gov/oncoarray/). The remaining included participants were from several GWAS, namely, BPC3, CaPS 1 and CaPS 2, iCOGS, NCI PEGASUS, and UK stage 1 and stage 2. More detailed information for characteristics of the cases and controls, including classifications for aggressiveness in cases has been described elsewhere. ${ }^{32}$ For example, the mean age ranged from 56 to 72 across the participating studies for cases, and ranged from 51 to 71 for controls. These genotype data were imputed using the 1000 Genomes Project data released in June 2014 as a reference. Summary statistics from logistic regression analyses were meta-analyzed using an inverse variance fixed effect approach further. Principal components and study-relevant covariates were controlled for in the OncoArray and each GWAS. Furthermore, country was stratified in the OncoArry, and study was stratified in the iCOGS analyses. It is worth noting that a key requirement of such type of analysis is that the dataset for estimating the SNP-exposure (in our case, SNP-glycan) associations do not have overlapped subjects with the dataset for estimating the SNP-outcome (in our case, SNP-prostate cancer) associations. ${ }^{34}$ This is for considering that to use datasets with overlapped samples for deriving the SNPexposure and SNP-outcome relationships may be prone to weak instrument bias towards the exposure-outcome estimate. ${ }^{34}$

The inverse variance weighted method (IVW), included in the MR-Base platform, ${ }^{35}$ was used to assess the associations of genetically predicted $\mathrm{N}$-glycan levels with prostate cancer risk. $^{26,36,37}$ In IVW method, $\sum_{i} \beta_{i}, G X^{*} \beta_{i}, G Y^{*} \sigma^{2_{i}}, G Y /\left(\sum_{i} \beta^{2_{i}}, G X^{*} \sigma^{2_{i}}, G Y\right) \quad$ and $1 /\left(\sum_{i} \beta^{2_{i}}, G X^{*} \sigma^{2_{i}}, G Y\right)^{0.5}$ were used to estimate the beta coefficient of the association between genetically predicted $\mathrm{N}$-glycan levels and prostate cancer risk, and the corresponding standard error, respectively. $\beta_{i, G X}$ here represents the beta coefficient of the association between $i$ th SNP and the glycan of interest reported in the literature; $\beta_{i, G Y}$ represents the beta coefficient of the association between $i$ th SNP and prostate cancer risk in the prostate cancer GWAS; and $\sigma_{i, G Y}$ represents the standard error of the association of SNP-prostate cancer. The association odds ratio (OR), confidence interval (CI), and $P$ value were estimated based on the calculated beta coefficient and standard error. A Bonferroni corrected threshold was used to determine significant associations. Given $80 \%$ power at the alpha of 0.05 , the minimal detectable ORs per standard deviation of genetically predicted $\mathrm{N}$-glycan levels ranged from 0.81 to 0.96 (from 1.04 to 1.23) based on power calculation using https://shiny.cnsge nomics.com $/ \mathrm{mRnd} / .^{38}$ 


\section{Results}

We evaluated the associations between $138 \mathrm{~N}$-glycan traits (of which 68 reflect total plasma N-glycome features, and 70 reflect IgG N-glycome) and prostate cancer risk. Detailed information of the genetic instruments used was shown in Supplementary Table 1. Aggregating independently associated variants together, $0.57 \%$ to $20.32 \%$ of the variation of $\mathrm{N}$-glycan traits can be explained by the corresponding instrument variants (Supplementary Table 1). The largest proportion of variation was explained for IGP29 (20.32\%). The smallest one was for IGP27 $(0.57 \%)$ with rs7621161 as the instrument.

The results for the associations between genetically predicted N-glycan levels and prostate cancer risk are shown in Table 1. Among all N-glycans tested, we identified three $\mathrm{N}$-glycans with genetically predicted levels to be associated with prostate cancer risk at $P \leq 3.62 \times 10^{-4}$, a Bonferroni-corrected significance level (0.05/138). Higher predicted levels of PGP18 (OR=1.29; 95\% CI, $\left.1.20-1.40 ; P=8.32 \times 10^{-9}\right)$ while lower predicted levels of PGP33 (OR=0.80; 95\% CI, 0.74-0.88; $P=1.33 \times 10^{-6}$ ) and PGP109 $(\mathrm{OR}=0.79 ; \quad 95 \%$ CI, $0.72-0.87 ; \quad P=$ $5.49 \times 10^{-7}$ ) were associated with an increased risk of prostate cancer (Table 1). The instruments for these three N-glycans are rs3115663 for PGP18, rs1866767 for PGP33, and rs11223780 and rs140053014 for PGP109 (Supplementary Table 1).

\section{Discussion}

Leveraging a large GWAS dataset for prostate cancer risk, we evaluated the relationship between genetically predicted plasma $\mathrm{N}$-glycan levels and prostate cancer risk. This study, to our knowledge, is the first study to characterize potential roles of $\mathrm{N}$-glycans in risk of prostate cancer using genetic instruments. We observed that lower predicted levels of PGP18 (The percentage of Man9 $\mathrm{N}$-glycan in total plasma glycans) as well as higher predicted levels of PGP33 (the percentage of A4G4S [3,3,3,3] 4 in total plasma glycans) and PGP109 (the ratio of disialylated and trisialylated trigalactosylated structures in total plasma glycans) were associated with lower risk of prostate cancer. Man9-mannosidase is a high mannose glycan, which is an alpha 1,2 specific enzyme located in the endoplasmic reticulum, and plays a key role in the processing of N-linked oligosaccharides. ${ }^{39}$ The A4G4S $[3,3,3,3] 4$ is a highly branched galactosylated sialylated glycan (attached to alpha1-acid protein - an acute-phase
Table I Associations Between Genetically Predicted Plasma N-Glycan Levels and Prostate Cancer Risk

\begin{tabular}{|c|c|c|}
\hline Trait & OR $(95 \% \mathrm{Cl})^{\mathrm{a}}$ & $P$ value $^{b}$ \\
\hline PGPI8 & $1.29(1.20-1.40)$ & $6.40 \times 10^{-11}$ \\
\hline PGPI09 & $0.79(0.72-0.87)$ & $5.49 \times 10^{-7}$ \\
\hline PGP33 & $0.80(0.74-0.88)$ & $1.33 \times 10^{-6}$ \\
\hline PGP2 & $0.92(0.87-0.98)$ & $5.47 \times 10^{-3}$ \\
\hline PGP3 & $0.95(0.88-1.02)$ & 0.18 \\
\hline PGP6 & $0.95(0.88-1.03)$ & 0.23 \\
\hline PGPI3 & $1.03(0.98-1.08)$ & 0.29 \\
\hline PGPI7 & $1.02(0.97-1.07)$ & 0.48 \\
\hline PGP20 & $1.01(0.95-1.08)$ & 0.68 \\
\hline PGP22 & $0.90(0.74-I . I)$ & 0.32 \\
\hline PGP23 & $0.97(0.91-1.02)$ & 0.26 \\
\hline PGP24 & I.0I (0.97-I.05) & 0.55 \\
\hline PGP25 & $1.00(0.94-1.06)$ & 0.95 \\
\hline PGP27 & $0.98(0.94-1.04)$ & 0.55 \\
\hline PGP28 & $0.97(0.89-1.05)$ & 0.43 \\
\hline PGP30 & 1.01 (0.97-1.04) & 0.69 \\
\hline PGP32 & $1.00(0.96-1.05)$ & 1.00 \\
\hline PGP34 & $1.00(0.95-1.06)$ & 0.96 \\
\hline PGP36 & $1.03(0.94-1.13)$ & 0.55 \\
\hline PGP37 & $0.98(0.91-1.07)$ & 0.71 \\
\hline PGP38 & $1.05(0.97-1.15)$ & 0.23 \\
\hline PGP39 & $0.97(0.89-1.05)$ & 0.44 \\
\hline PGP40 & $1.05(0.97-1.15)$ & 0.23 \\
\hline PGP4I & $1.03(0.98-1.09)$ & 0.28 \\
\hline PGP42 & 1.01 (0.9-1.14) & 0.82 \\
\hline PGP43 & $0.98(0.92-1.04)$ & 0.55 \\
\hline PGP45 & $1.07(0.96-1.2)$ & 0.23 \\
\hline PGP46 & $0.89(0.74-1.07)$ & 0.23 \\
\hline PGP47 & $1.02(0.96-1.08)$ & 0.54 \\
\hline PGP50 & $1.04(0.95-1.14)$ & 0.43 \\
\hline PGP5I & $1.03(0.95-1.12)$ & 0.43 \\
\hline PGP52 & $1.03(0.96-1.11)$ & 0.43 \\
\hline \multirow[t]{4}{*}{ PGP56 } & $1.02(0.98-1.06)$ & 0.37 \\
\hline & $1.02(0.98-1.05)$ & 0.43 \\
\hline & $1.02(0.97-1.07)$ & 0.52 \\
\hline & $1.09(0.82-1.46)$ & 0.67 \\
\hline PGP57 & $1.01(0.97-1.06)$ & 0.57 \\
\hline PGP59 & $0.94(0.87-1.01)$ & 0.09 \\
\hline PGP60 & $1.00(0.93-1.08)$ & 0.97 \\
\hline PGP6I & $1.06(0.97-1.16)$ & 0.23 \\
\hline PGP62 & $1.01(0.93-1.1)$ & 0.80 \\
\hline PGP63 & $0.95(0.88-1.03)$ & 0.23 \\
\hline PGP67 & $\mathrm{I} .05(0.99-1.1 \mathrm{I})$ & 0.08 \\
\hline PGP70 & $0.93(0.85-1.01)$ & 0.09 \\
\hline PGP72 & $1.06(0.99-1.14)$ & 0.09 \\
\hline PGP74 & $0.99(0.93-1.05)$ & 0.74 \\
\hline PGP78 & $1.04(0.97-1.12)$ & 0.23 \\
\hline PGP79 & $1.08(0.99-1.18)$ & 0.09 \\
\hline PGP80 & $0.95(0.88-1.03)$ & 0.23 \\
\hline PGP82 & $0.96(0.89-1.03)$ & 0.23 \\
\hline
\end{tabular}

(Continued) 
Table I (Continued).

\begin{tabular}{|c|c|c|}
\hline Trait & OR $(95 \% \mathrm{CI})^{\mathrm{a}}$ & $P$ value $^{b}$ \\
\hline PGP83 & $0.95(0.87-1.03)$ & 0.23 \\
\hline PGP84 & $0.95(0.86-1.04)$ & 0.23 \\
\hline PGP85 & $0.95(0.87-1.03)$ & 0.23 \\
\hline PGP86 & $1.05(0.97-1.15)$ & 0.23 \\
\hline PGP87 & $1.00(0.92-1.09)$ & 0.97 \\
\hline PGP88 & $0.95(0.87-1.03)$ & 0.23 \\
\hline PGP89 & $0.95(0.87-1.03)$ & 0.23 \\
\hline PGP90 & $1.05(0.97-1.14)$ & 0.23 \\
\hline PGP92 & $0.96(0.88-1.04)$ & 0.34 \\
\hline PGP99 & $0.94(0.84-1.05)$ & 0.30 \\
\hline PGPI02 & $0.97(0.91-1.04)$ & 0.43 \\
\hline PGPI03 & $1.02(0.96-1.09)$ & 0.55 \\
\hline PGPI05 & $0.98(0.92-1.04)$ & 0.55 \\
\hline PGPI06 & $1.02(0.96-1.08)$ & 0.55 \\
\hline PGPI07 & $0.95(0.88-1.03)$ & 0.23 \\
\hline PGPIIO & $0.93(0.86-1.02)$ & 0.12 \\
\hline PGPII2 & $1.00(0.95-1.05)$ & 0.99 \\
\hline IGP2 & $0.98(0.93-1.03)$ & 0.43 \\
\hline IGP3 & $0.98(0.87-\mathrm{I} . \mathrm{II})$ & 0.75 \\
\hline IGP5 & $1.03(0.96-1.1 \mathrm{I})$ & 0.40 \\
\hline IGP6 & $0.97(0.87-1.1)$ & 0.66 \\
\hline IGP7 & $1.04(0.94-1.16)$ & 0.45 \\
\hline IGP8 & 1.01 (0.94-I.08) & 0.88 \\
\hline IGP9 & 1.01 (0.96-I.06) & 0.66 \\
\hline IGPIO & I.0I (0.94-I.09) & 0.78 \\
\hline IGPII & $0.97(0.91-1.03)$ & 0.36 \\
\hline IGPI 2 & $0.99(0.92-1.07)$ & 0.84 \\
\hline IGPI 3 & $0.97(0.91-1.03)$ & 0.28 \\
\hline IGPI4 & $0.96(0.91-1.02)$ & 0.17 \\
\hline IGPI5 & $1.04(0.96-1.13)$ & 0.33 \\
\hline IGPI7 & $1.04(0.99-1.1)$ & 0.14 \\
\hline IGPI8 & $0.97(0.87-1.07)$ & 0.55 \\
\hline IGPI9 & $0.98(0.85-1.13)$ & 0.78 \\
\hline IGP2I & $0.96(0.87-1.07)$ & 0.49 \\
\hline IGP22 & $1.03(0.97-1.09)$ & 0.32 \\
\hline IGP23 & $1.05(0.98-1.13)$ & 0.18 \\
\hline IGP24 & $1.01(0.96-1.06)$ & 0.72 \\
\hline IGP25 & $1.10(0.96-1.26)$ & 0.18 \\
\hline IGP26 & $1.02(0.97-1.08)$ & 0.41 \\
\hline IGP27 & I.II (0.95-I.28) & 0.18 \\
\hline IGP28 & $1.05(0.94-1.16)$ & 0.40 \\
\hline IGP29 & I.0I (0.97-I.05) & 0.70 \\
\hline IGP30 & $1.02(0.96-1.09)$ & 0.51 \\
\hline IGP3| & $1.00(0.93-1.07)$ & 0.93 \\
\hline IGP32 & $1.03(0.99-1.07)$ & 0.19 \\
\hline IGP34 & $1.00(0.93-1.08)$ & 0.96 \\
\hline IGP35 & $0.97(0.94-1.01)$ & 0.18 \\
\hline IGP36 & $1.00(0.8-1.24)$ & 0.98 \\
\hline IGP37 & $0.98(0.86-|| 2)$. & 0.77 \\
\hline IGP38 & $0.98(0.86-1.1 \mathrm{I})$ & 0.76 \\
\hline
\end{tabular}

(Continued)
Table I (Continued).

\begin{tabular}{|c|c|c|}
\hline Trait & OR $(95 \% \mathrm{Cl})^{\mathrm{a}}$ & $P$ value $^{b}$ \\
\hline IGP39 & $1.01(0.97-1.05)$ & 0.66 \\
\hline IGP40 & $1.00(0.96-1.04)$ & 0.93 \\
\hline IGP42 & $0.98(0.93-1.03)$ & $0.4 I$ \\
\hline IGP43 & $0.98(0.86-1.11)$ & 0.75 \\
\hline IGP45 & $1.03(0.96-1.1)$ & 0.42 \\
\hline IGP46 & $0.97(0.87-I . I)$ & 0.66 \\
\hline IGP47 & I.0I (0.93-I.II) & 0.76 \\
\hline IGP48 & $1.08(0.9-1.28)$ & 0.41 \\
\hline IGP49 & $1.01(0.96-1.06)$ & 0.66 \\
\hline IGP50 & I.0I (0.94-I.09) & 0.77 \\
\hline IGP5| & $0.97(0.9-1.04)$ & 0.40 \\
\hline IGP52 & $1.01(0.94-1.08)$ & 0.84 \\
\hline IGP53 & $0.97(0.91-1.04)$ & 0.38 \\
\hline \multirow[t]{2}{*}{ IGP54 } & $0.97(0.89-1.05)$ & 0.41 \\
\hline & $1.00(0.92-1.09)$ & 0.98 \\
\hline IGP55 & $0.96(0.84-1.09)$ & 0.50 \\
\hline IGP57 & $0.97(0.9-1.04)$ & 0.34 \\
\hline IGP58 & $1.03(0.98-1.08)$ & 0.31 \\
\hline IGP59 & $1.02(0.98-1.07)$ & 0.32 \\
\hline IGP60 & $1.03(0.98-1.08)$ & 0.31 \\
\hline IGP6| & $1.03(0.98-1.08)$ & 0.27 \\
\hline IGP62 & $0.98(0.93-1.04)$ & 0.60 \\
\hline IGP63 & $1.00(0.95-1.06)$ & 0.98 \\
\hline IGP64 & $0.99(0.94-1.05)$ & 0.76 \\
\hline IGP65 & $1.01(0.95-1.06)$ & 0.79 \\
\hline IGP66 & $1.01(0.96-1.07)$ & 0.60 \\
\hline IGP67 & $1.00(0.95-1.05)$ & 0.92 \\
\hline IGP68 & $1.01(0.96-1.06)$ & 0.76 \\
\hline IGP69 & $1.00(0.95-1.05)$ & 0.86 \\
\hline IGP70 & $1.01(0.96-1.07)$ & 0.60 \\
\hline IGP7| & $1.01(0.96-1.07)$ & 0.60 \\
\hline IGP72 & $0.98(0.94-1.03)$ & 0.52 \\
\hline IGP73 & $1.01(0.94-1.08)$ & 0.85 \\
\hline IGP74 & $1.00(0.95-1.04)$ & 0.88 \\
\hline IGP75 & $1.00(0.95-1.04)$ & 0.89 \\
\hline IGP76 & $1.00(0.95-1.05)$ & 0.98 \\
\hline IGP77 & $0.99(0.92-1.06)$ & 0.72 \\
\hline
\end{tabular}

Notes: ${ }^{a} O R$ represents odds ratio per one standard deviation increase in genetically predicted levels of $\mathrm{N}$-glycan; $\mathrm{Cl}$ represents confidence interval of the odds ratio; ${ }^{b} P$ value: Derived from association analyses of 79,194 cases and 61,112 controls.

protein, ${ }^{40}$ known to be associated with chronic low-grade inflammation. $^{41,42}$ The observed inverse association of A4G4S[3,3,3,3]4 with prostate cancer risk is not entirely consistent with known knowledge of A4G4S[3,3,3,3]4 with higher level of low-grade inflammation and future work is needed to better understand the relationship between these two. Our work provides new information to understand the relationship between $\mathrm{N}$-glycans and 
prostate cancer. Due to the potential pleiotropy bias, additional work is needed to better understand whether changes in $\mathrm{N}$-glycosylations could influence the risk of prostate cancer.

Recently, studies suggested that changes to N-glycans such as the branching of complex biantennary glycans to triantennary and tetraantennary structures and the emergence of cryptic N-glycans could be related to prostate cancer. ${ }^{12,43-46}$ Kawahara et al found that a panel of 56 intact $\mathrm{N}$-glycopeptides could discriminate prostate cancer from benign prostatic hyperplasia. ${ }^{43}$ Several other studies observed that changes to branched N-glycans could also help distinguish prostate cancer from benign prostatic hyperplasia. ${ }^{44,45}$ Moreover, it was identified that tetraantennary and tetrasialylated N-glycans could potentially serve as biomarkers to improve the stratification of patients with indolent and aggressive prostate cancer. ${ }^{46}$ Matsumoto et al identified that castration-resistant prostate cancer (CRPC) patients had significantly higher levels of terminal $\alpha-2.3$ sialylated glycan, $\alpha-2.6$ sialylated glycan, and terminal galactose while lower levels of branchedLacNAc structure compared with non-CRPC patients. ${ }^{47}$ Ishibashi et al found that tri- and tetra-antennary $\mathrm{N}$-glycans were significantly higher in CRPC patients than other groups including healthy volunteers, subjects with benign prostatic hyperplasia, early-stage prostate cancer patients, as well as prostate cancer patients with androgen deprivation therapy. ${ }^{48}$ Also, the expression of $\mathrm{N}$-glycan branching enzyme genes (MGAT1, MGAT2, $M G A T 4 B, M G A T 5 A$, and MGAT5B) were significantly higher in the CRPC-like cell lines DU145 and PC-3 compared with androgen-dependent LNCaP cells and normal prostate epithelial RWPE-1 cells. In addition, IgG antibodies targeting the Man9- or Tri-/m-II-autoantigens were detectable and these antibody activities were selectively increased in prostate cancer patients compared with benign prostatic hyperplasia patients. ${ }^{49}$ Ishikawa et al developed an automated system for measuring cancer-associated $\alpha$ 2,3-linked sialyl N-Glycan-carrying prostate-specific antigen (S2, 3PSA). The area under the curve (AUC) for the S2,3PSA ratio was 0.83 which was much higher than conventional PSA testing (AUC $=0.51$ ), in their analyses differentiating 50 prostate cancer patients from 50 benign prostatic hyperplasia patients. ${ }^{50}$ Llop et al conducted a study to assess the core fucosylation and the sialic acid linkage of PSA N-glycans in serum samples from 29 benign prostatic hyperplasia patients and 44 prostate cancer patients with different degrees of aggressiveness. They found significant increased levels of the $\alpha-2,3$-sialic acid and decreased levels of the core fucose percentage of PSA $\mathrm{N}$-glycans in aggressive prostate cancer compared with benign prostatic hyperplasia and nonaggressive prostate cancer. ${ }^{51}$ As the precursors, cores, and internal sequences of $\mathrm{N}$-glycans, cryptic $\mathrm{N}$-glycans are reported to be closely related to development of prostate cancer. ${ }^{21,49,52,53}$ Wang et al reported the detection of cryptic N-glycan Man9 in the serum of prostate cancer patients, and its antibodies could help differentiate high-grade prostate cancer. ${ }^{54}$ It would be interesting to investigate whether specific $\mathrm{N}$-glycans could provide useful information for prognosis of advanced prostate cancer patients such as CRPC according to therapy. ${ }^{55-57}$ Beyond the scope of glycans, research has supported potential roles of additional markers in risk prediction of prostate cancer. For example, the prostate health index (PHI) was reported as a better predictor of positive first biopsy in men with total PSA "gray" levels of $2-10 \mathrm{ng} / \mathrm{mL}$ compared with \%fPSA (free to total PSA ratio $\times 100) .{ }^{58}$ Future work assessing whether a combination of $\mathrm{N}$-glycans and $\mathrm{PHI}$ can further improve the prediction may be warranted.

The proportions of variance of $\mathrm{N}$-glycans that can be explained by the summed GWAS-identified genetic variants are relatively high for many of the assessed $\mathrm{N}$-glycans, suggesting that many of the instrumental variables used in this study are relatively strong. The main association analysis of our study leveraging a large number of prostate cancer cases and controls provided high statistical power to detect the glycan-prostate cancer associations. The design of using genetic instruments could reduce selection bias, potential confounding, and reverse causation bias that are commonly imbedded in conventional observational studies. On the other hand, there are potential limitations of our study. Given the correlated nature of the $\mathrm{N}$-glycans themselves and that many $\mathrm{N}$-glycans share genetic loci, pleiotropy will be a major concern. While observed association of a variant with two traits could be because of pleiotropy of a single functional variant, it could as well be because of linkage disequilibrium between two distinct functional variants. Thus, our findings should be taken with caution and further studies are needed to validate them. Second, our analysis may be constrained by the variants identified in previous GWAS of plasma levels of N-glycans. In the study of Sharapov et al, the discovery analysis was based on TwinsUK dataset, which consisted of $89 \%$ females and $11 \%$ males. On the other hand, prior to GWAS, the glycan traits were adjusted 
or age and sex. Furthermore, as described in the original paper, for a majority of the GWAS identified associations, they were further replicated in three additional datasets (PainOR, QMDiab, and SOCCS), which contain relatively higher proportions of males $(45 \%, 50 \%$, and $54 \%$, respectively). It is expected that if we only focus on datasets of males in these studies for determining glycan associated variants, the statistical power would be relatively low. Considering that the replication analysis using data involving much higher proportions of males largely support the variant-glycan associations identified in the discovery analysis, we think that the current strategy using instruments based on analyses of combined sex may be more appropriate. In the Klaric et al, the discovery analyses were performed in four cohorts, CROATIA-Korcula (38\% males), CROATIA-Vis (46\% males), ORCADES (41\% males), and TwinsUK (all females). The glycan traits were corrected for age, sex, cohort-specific covariates and cryptic relatedness before analyses. It is expected that additional N-glycan biomarkers could be identified using newly identified genetic variants (preferably in studies of males only with sufficient sample sizes) in the future. Furthermore, future work using comprehensive genetic prediction models of $\mathrm{N}$-glycans aggregating effects of multiple variants could further improve the statistical power with increased variance of $\mathrm{N}$-glycan levels that could be explained by genetic instruments. Third, the present work primarily focuses on studying prostate cancer risk comparing cases versus controls. Future work considering clinical and pathological features of prostate cancer (such as stage and Gleason score) would be needed to identify glycans that are potentially related to prostate cancer aggressiveness. It would also be useful to incorporate family history information into analyses to differentiate glycans playing a different role in familiar versus sporadic prostate cancer. Further studies are also needed to verify our findings with directly measured levels of these glycans. Functional investigations are also warranted to understand the biological roles of the identified $\mathrm{N}$-glycans in prostate tumorigenesis.

\section{Conclusions}

In this large-scale study, we identified three N-glycans with genetically predicted levels in plasma to be associated with prostate cancer risk. However, potential biases could have influenced these observed associations. Future work is needed to better characterize the relationship between $\mathrm{N}$-glycans and prostate cancer.

\section{Summary}

Prostate cancer is the second most commonly diagnosed cancer among males worldwide. Prostate-specific antigen screening is controversial due to the lack of a clear cutoff point for high sensitivity and specificity, and unclear benefit in reducing mortality in specific populations. Therefore, there are critical needs for better understanding the etiology and identifying effective biomarkers to improve the risk assessment of prostate cancer. The purpose of this study is to better characterize the relationship between glycosylation and prostate cancer, using genetic instruments. In this work, we identified several N-glycan biomarker candidates for prostate cancer risk. It not only provides new data regarding novel candidate risk biomarkers for prostate cancer but also demonstrates the utility of integrating genomics and glycomics data in biomarker research.

\section{Data Availability}

For the prostate cancer GWAS data in the PRACTICAL consortium, the OncoArray genotype data and relevant covariate information (ie, ethnicity, country, principal components, etc.) are available in dbGAP (Accession \#: phs001391.v1.p1). In total, 47 of the 52 OncoArray studies, encompassing nearly $90 \%$ of the individual samples, are available. The previous meta-analysis summary results and genotype data are currently available in dbGAP (Accession \#: phs001081.v1.p1). The overall meta summary association statistics as described in Schumacher et al 2018 are available at http://practical.icr.ac.uk/blog/? page $\mathrm{id}=8164$.

\section{Acknowledgments}

We would like to thank Yurii Aulchenko, Azra Frkatović and Gordan Lauc for their help for our study. This study is supported by the University of Hawaii Cancer Center. Duo Liu is partially supported by the Harbin Medical University Cancer Hospital, Chinese Medical Association Clinical Pharmacy Branch-Wu Jieping Medical Foundation Research Fund (No. 320.6750.19090-41) and Youth science of Harbin Medical University Cancer Hospital (Grant no. BJQN2018-03). The work of SSH and ET are supported by a grant from the Russian Science Foundation (RSF) No. 19-15-00115. We thank The PRACTICAL consortium, CRUK, BPC3, CAPS, PEGASUS for making the GWAS summary statistics to be publically available. The Prostate cancer genome-wide 
association analyses are supported by the Canadian Institutes of Health Research, European Commission's Seventh Framework Programme grant agreement $n^{\circ}$ 223175 (HEALTH-F2-2009-223175), Cancer Research UK Grants C5047/A7357, C1287/A10118, C1287/ A16563, C5047/A3354, C5047/A10692, C16913/A6135, and The National Institute of Health (NIH) Cancer PostCancer GWAS initiative grant: No. 1 U19 CA 148537-01 (the GAMe-ON initiative). We would also like to thank the following for funding support: The Institute of Cancer Research and The Everyman Campaign, The Prostate Cancer Research Foundation, Prostate Research Campaign UK (now PCUK), The Orchid Cancer Appeal, Rosetrees Trust, The National Cancer Research Network UK, The National Cancer Research Institute (NCRI) UK. We are grateful for support of NIHR funding to the NIHR Biomedical Research Centre at The Institute of Cancer Research and The Royal Marsden NHS Foundation Trust. The Prostate Cancer Program of Cancer Council Victoria also acknowledge grant support from The National Health and Medical Research Council, Australia (126402, 209057, 251533, 396414, 450104, 504700, 504702, 504715, 623204, 940394, 614296,), VicHealth, Cancer Council Victoria, The Prostate Cancer Foundation of Australia, The Whitten Foundation, PricewaterhouseCoopers, and Tattersall's. EAO, DMK, and EMK acknowledge the Intramural Program of the National Human Genome Research Institute for their support. Genotyping of the OncoArray was funded by the US National Institutes of Health (NIH) [U19 CA 148537 for ELucidating Loci Involved in Prostate cancer SuscEptibility (ELLIPSE) project and X01HG007492 to the Center for Inherited Disease Research (CIDR) under contract number HHSN268201200008I] and by Cancer Research UK grant A8197/A16565. Additional analytic support was provided by NIH NCI U01 CA188392 (PI: Schumacher). Funding for the iCOGS infrastructure came from: the European Community's Seventh Framework Programme under grant agreement $\mathrm{n}^{\circ} 223175$ (HEALTHF2-2009-223175) (COGS), Cancer Research UK (C1287/ A10118, C1287/A 10710, C12292/A11174, C1281/ A12014, C5047/A8384, C5047/A15007, C5047/A10692, C8197/A16565), the National Institutes of Health (CA128978) and Post-Cancer GWAS initiative (1U19 CA148537, 1U19 CA148065 and 1U19 CA148112 - the GAM $\times 10$-ON initiative), the Department of Defence (W81XWH-10-1-0341), the Canadian Institutes of Health Research (CIHR) for the CIHR Team in Familial Risks of
Breast Cancer, Komen Foundation for the Cure, the Breast Cancer Research Foundation, and the Ovarian Cancer Research Fund. The BPC3 was supported by the US National Institutes of Health, National Cancer Institute (cooperative agreements U01-CA98233 to D.J.H., U01CA98710 to S.M.G., U01-CA98216 toE.R., and U01CA98758 to B.E.H., and Intramural Research Program of NIH/National Cancer Institute, Division of Cancer Epidemiology and Genetics). CAPS GWAS study was supported by the Swedish Cancer Foundation (grant no 09-0677, 11-484, 12-823), the Cancer Risk Prediction Center (CRisP; www.crispcenter.org), a Linneus Centre (Contract ID 70867902) financed by the Swedish Research Council, Swedish Research Council (grant no K2010-70X-20430-04-3, 2014-2269). PEGASUS was supported by the Intramural Research Program, Division of Cancer Epidemiology and Genetics, National Cancer Institute, National Institutes of Health.

\section{Disclosure}

The authors declare no potential conflicts of interest.

\section{References}

1. Bray F, Ferlay J, Soerjomataram I, Siegel RL, Torre LA, Jemal A. Global cancer statistics 2018: GLOBOCAN estimates of incidence and mortality worldwide for 36 cancers in 185 countries. CA Cancer J Clin. 2018;68:394-424.

2. Siegel RL, Miller KD, Jemal A. Cancer statistics, 2020. CA Cancer J Clin. 2020;70:7-30.

3. Litwin MS, Tan H-J. The diagnosis and treatment of prostate cancer: a review. JAMA. 2017;317(24):2532-2542. doi:10.1001/ jama.2017.7248

4. Pezaro C, Woo HH, Davis ID. Prostate cancer: measuring PSA. Intern Med J. 2014;44(5):433-440. doi:10.1111/imj.12407

5. Thompson IM, Ankerst DP, Chi C, et al. Operating characteristics of prostate-specific antigen in men with an initial PSA level of $3.0 \mathrm{ng} /$ $\mathrm{mL}$ or lower. JAMA. 2005;294(1):66-70. doi:10.1001/jama.294.1.66

6. Thompson IM, Pauler DK, Goodman PJ, et al. Prevalence of prostate cancer among men with a prostate-specific antigen level $\leq 4.0 \mathrm{ng}$ per milliliter. $N$ Engl J Med. 2004;350(22):2239-2246. doi:10.1056/ NEJMoa031918

7. Parekh DJ, Ankerst DP, Troyer D, Srivastava S, Thompson IM. Biomarkers for prostate cancer detection. $J$ Urol. 2007;178:2252-2259. doi:10.1016/j.juro.2007.08.055

8. Schröder FH, Hugosson J, Roobol MJ, et al. Screening and prostate cancer mortality: results of the European Randomised Study of Screening for Prostate Cancer (ERSPC) at 13 years of follow-up. Lancet. 2014;384(9959):2027-2035. doi:10.1016/S0140-6736(14) 60525-0

9. Schröder FH, Hugosson J, Roobol MJ, et al. Screening and prostate-cancer mortality in a randomized European study. $N$ Engl $J$ Med. 2009;360(13):1320-1328. doi:10.1056/NEJMoa0810084

10. Andriole GL, Crawford ED, Grubb RL, et al. Mortality results from a randomized prostate-cancer screening trial. $N$ Engl $J$ Med. 2009;360(13):1310-1319. doi:10.1056/NEJMoa0810696

11. Munkley J. The glycosylation landscape of pancreatic cancer. Oncol Lett. 2019;17:2569-2575. 
12. Pinho SS, Reis CA. Glycosylation in cancer: mechanisms and clinical implications. Nat Rev Cancer. 2015;15(9):540-555. doi:10.1038/ nrc3982

13. Demetriou M, Nabi IR, Coppolino M, Dedhar S, Dennis JW. Reduced contact-inhibition and substratum adhesion in epithelial cells expressing GlcNAc-transferase V. J Cell Biol. 1995;130 (2):383-392. doi:10.1083/jcb.130.2.383

14. Seberger PJ, Chaney WG. Control of metastasis by Asn-linked, $\beta 1-6$ branched oligosaccharides in mouse mammary cancer cells. Glycobiology. 1999;9:235-241. doi:10.1093/glycob/9.3.235

15. Granovsky M, Fata J, Pawling J, Muller WJ, Khokha R, Dennis JW. Suppression of tumor growth and metastasis in Mgat5-deficient mice. Nat Med. 2000;6:306-312. doi:10.1038/73163

16. Takahashi M, Kuroki Y, Ohtsubo K, Taniguchi N. Core fucose and bisecting GlcNAc, the direct modifiers of the N-glycan core: their functions and target proteins. Carbohydr Res. 2009;344:1387-1390. doi:10.1016/j.carres.2009.04.031

17. Yoshimura M, Nishikawa A, Ihara Y, Taniguchi S, Taniguchi N. Suppression of lung metastasis of B16 mouse melanoma by $\mathrm{N}$-acetylglucosaminyltransferase III gene transfection. Proc Natl Acad Sci. 1995;92:8754-8758. doi:10.1073/pnas.92.19.8754

18. Zhao Y, Sato Y, Isaji $\mathrm{T}$, et al. Branched N-glycans regulate the biological functions of integrins and cadherins. FEBS J. 2008;275:1939-1948. doi:10.1111/j.1742-4658.2008.06346.x

19. Scott E, Munkley J. Glycans as biomarkers in prostate cancer. Int J Mol Sci. 2019;20(6):1389. doi:10.3390/ijms20061389

20. Stanley P, Schachter H, Taniguchi N. N-Glycans. In: Varki A, Cummings RD, Esko JD, et al, eds. Essentials of Glycobiology. 2nd edition. Cold Spring Harbor (NY): Cold Spring Harbor Laboratory Press; 2009. Chapter 8.

21. Lange T, Ullrich S, Müller I, et al. Human prostate cancer in a clinically relevant xenograft mouse model: identification of $\beta(1,6)$-branched oligosaccharides as a marker of tumor progression. Clin Cancer Res. 2012;18(5):13641373. doi:10.1158/1078-0432.CCR-11-2900

22. Doherty M, Theodoratou E, Walsh I, et al. Plasma N-glycans in colorectal cancer risk. Sci Rep. 2018;8:1-12. doi:10.1038/s41598018-26805-7

23. Lawlor DA, Harbord RM, Sterne JA, Timpson N, Davey Smith G. Mendelian randomization: using genes as instruments for making causal inferences in epidemiology. Stat Med. 2008;27 (8):1133-1163. doi:10.1002/sim.3034

24. Teumer A. Common methods for performing Mendelian randomization. Front Cardiovasc Med. 2018;5:51. doi:10.3389/ fcvm.2018.00051

25. Swerdlow DI, Kuchenbaecker KB, Shah S, et al. Selecting instruments for Mendelian randomization in the wake of genome-wide association studies. Int $J$ Epidemiol. 2016;45(5):1600-1616. doi:10.1093/ije/dyw088

26. Sharapov SZ, Tsepilov YA, Klaric L, et al. Defining the genetic control of human blood plasma N-glycome using genome-wide association study. Hum Mol Genet. 2019;28:2062-2077.

27. Klarić L, Tsepilov YA, Stanton CM, et al. Glycosylation of immunoglobulin $\mathrm{G}$ is regulated by a large network of genes pleiotropic with inflammatory diseases. Sci Advan. 2020;6:eaax0301. doi:10.1126/sciadv.aax0301

28. Benedetti E, Pučić-Baković M, Keser T, et al. Network inference from glycoproteomics data reveals new reactions in the IgG glycosylation pathway. Nat Commun. 2017;8:1483. doi:10.1038/s41467017-01525-0

29. Akmačić IT, Ugrina I, Štambuk J, et al. High-throughput glycomics: optimization of sample preparation. Biochemistry. 2015;80 (7):934-942. doi:10.1134/S0006297915070123

30. Lauc G, Huffman JE, Pučić M, et al. Loci associated with $\mathrm{N}$-glycosylation of human immunoglobulin $\mathrm{G}$ show pleiotropy with autoimmune diseases and haematological cancers. PLoS Genet. 2013;9(1):e1003225. doi:10.1371/journal.pgen.1003225
31. Wu L, Wang J, Cai Q, et al. Identification of novel susceptibility loci and genes for prostate cancer risk: a transcriptome-wide association study in over 140,000 European descendants. Cancer Res. 2019;79 (13):3192-3204. doi:10.1158/0008-5472.CAN-18-3536

32. Schumacher FR, Al Olama AA, Berndt SI, et al. Association analyses of more than 140,000 men identify 63 new prostate cancer susceptibility loci. Nat Genet. 2018;50(7):928. doi:10.1038/s41588-0180142-8

33. Wu L, Yang Y, Guo X, et al. An integrative multi-omics analysis to identify candidate DNA methylation biomarkers related to prostate cancer risk. Nat Commun. 2020;11(1):3905. doi:10.1038/s41467-02017673-9

34. Hartwig FP, Davies NM, Hemani G, Davey Smith G. Two-sample Mendelian randomization: avoiding the downsides of a powerful, widely applicable but potentially fallible technique. Int J Epidemiol. 2016;45(6):1717-1726. doi:10.1093/ije/dyx028

35. Hemani G, Zheng J, Elsworth B, et al. The MR-Base platform supports systematic causal inference across the human phenome. Elife. 2018;7:e34408. doi:10.7554/eLife.34408

36. Wu L, Shu X, Bao J, et al. PRACTICAL, CRUK, BPC3, CAPS, PEGASUS Consortia. Analysis of over 140,000 European descendants identifies genetically predicted blood protein biomarkers associated with prostate cancer risk. Cancer Res. 2019;79 (18):4592-4598. doi:10.1158/0008-5472.CAN-18-3997

37. Zhu J, Shu X, Guo X, et al. Associations between genetically predicted blood protein biomarkers and pancreatic cancer risk. Cancer Epidemiol Prev Biomarkers. 2020;29(7):1501-1508. doi:10.1158/ 1055-9965.EPI-20-0091

38. Brion M-JA, Shakhbazov K, Visscher PM. Calculating statistical power in Mendelian randomization studies. Int $J$ Epidemiol. 2013;42(5):1497-1501. doi:10.1093/ije/dyt179

39. Bause E, Breuer W, Schweden J, Roeser R, Geyer R. Effect of substrate structure on the activity of Man9-mannosidase from pig liver involved in N-linked oligosaccharide processing. Eur $J$ Biochem. 1992;208(2):451-457. doi:10.1111/j.1432-1033.1992. tb17207.x

40. Clerc F, Reiding KR, Jansen BC, Kammeijer GS, Bondt A, Wuhrer M. Human plasma protein N-glycosylation. Glycoconj J. 2016;33:309-343.

41. Novokmet M, Lukić E, Vučković F, et al. Changes in IgG and total plasma protein glycomes in acute systemic inflammation. Sci Rep. 2014;4(1):4347. doi:10.1038/srep04347

42. Schmidt MI, Duncan BB, Sharrett AR, et al. Markers of inflammation and prediction of diabetes mellitus in adults (Atherosclerosis Risk in Communities study): a cohort study. Lancet. 1999;353 (9165):1649-1652. doi:10.1016/S0140-6736(99)01046-6

43. Kawahara R, Ortega F, Rosa-Fernandes L, et al. Distinct urinary glycoprotein signatures in prostate cancer patients. Oncotarget. 2018;9(69):33077. doi:10.18632/oncotarget.26005

44. Bhat G, Hothpet V-R, Lin M-F, Cheng P-W. Shifted Golgi targeting of glycosyltransferases and $\alpha$-mannosidase IA from giantin to GM130-GRASP65 results in formation of high mannose N-glycans in aggressive prostate cancer cells. Biochimica et Biophysica Acta. 2017;1861(11):2891-2901. doi:10.1016/j.bbagen.2017.08.006

45. Saldova R, Fan Y, Fitzpatrick JM, Watson RWG, Rudd PM. Core fucosylation and $\alpha 2-3$ sialylation in serum $\mathrm{N}$-glycome is significantly increased in prostate cancer comparing to benign prostate hyperplasia. Glycobiology. 2011;21(2):195-205. doi:10.1093/glycob/cwq147

46. Murphy K, Murphy BT, Boyce S, et al. Integrating biomarkers across omic platforms: an approach to improve stratification of patients with indolent and aggressive prostate cancer. Mol Oncol. 2018;12 (9):1513-1525. doi:10.1002/1878-0261.12348

47. Matsumoto T, Hatakeyama S, Yoneyama T, et al. Serum N-glycan profiling is a potential biomarker for castration-resistant prostate cancer. Sci Rep. 2019;9:1-8. doi:10.1038/s41598-019-53384-y 
48. Ishibashi Y, Tobisawa Y, Hatakeyama S, et al. Serum tri- and tetra-antennary $\mathrm{N}$-glycan is a potential predictive biomarker for castration-resistant prostate cancer. Prostate. 2014;74 (15):1521-1529. doi:10.1002/pros.22869

49. Wang D. N-glycan cryptic antigens as active immunological targets in prostate cancer patients. J Proteomics Bioinform. 2012;5(04):090. doi:10.4172/jpb.1000218

50. Ishikawa T, Yoneyama T, Tobisawa Y, et al. An automated micrototal immunoassay system for measuring cancer-associated $\alpha 2$, 3-linked sialyl N-glycan-carrying prostate-specific antigen may improve the accuracy of prostate cancer diagnosis. Int $J$ Mol Sci. 2017;18(2):470. doi:10.3390/ijms18020470

51. Llop E, Ferrer-Batallé M, Barrabés S, et al. Improvement of prostate cancer diagnosis by detecting PSA glycosylation-specific changes. Theranostics. 2016;6(8):1190. doi:10.7150/thno.15226

52. Munkley J, Mills IG, Elliott DJ. The role of glycans in the development and progression of prostate cancer. Nat Rev Urol. 2016;13 (6):324. doi:10.1038/nrurol.2016.65

53. Wang D, Herzenberg LA, Peehl DM, Herzenberg LA Prostate cancer glycan markers and autoantibody signatures. Google Patents. 2011.

54. Wang D, Dafik L, Nolley R, et al. Anti-oligomannose antibodies as potential serum biomarkers of aggressive prostate cancer. Drug Dev Res. 2013;74(2):65-80. doi:10.1002/ddr.21063
55. Lorenzo GD, Zappavigna S, Crocetto F, et al. Assessment of total, PTEN -, and AR-V7 + circulating tumor cell count by flow cytometry in patients with metastatic castration-resistant prostate cancer receiving enzalutamide. Clin Genitourin Cancer. 2021. doi:10.1016/j. clgc.2021.03.021

56. Ferro M, Lucarelli G, Crocetto F, et al. First-line systemic therapy for metastatic castration-sensitive prostate cancer: an updated systematic review with novel findings. Crit Rev Oncol Hematol. 2021;157:103198. doi:10.1016/j.critrevonc.2020.103198

57. Buonerba C, Ferro M, Dolce P, et al. Predictors of efficacy of androgen-receptor-axis-targeted therapies in patients with metastatic castration-sensitive prostate cancer: a systematic review and meta-analysis. Crit Rev Oncol Hematol. 2020;151:102992. doi:10.1016/j.critrevonc.2020.102992

58. Bruzzese D, Mazzarella C, Ferro M, et al. Prostate health index vs percent free prostate-specific antigen for prostate cancer detection in men with "gray" prostate-specific antigen levels at first biopsy: systematic review and meta-analysis. Transl Res. 2014;164 (6):444-451. doi:10.1016/j.trs1.2014.06.006
Pharmacogenomics and Personalized Medicine

\section{Publish your work in this journal}

Pharmacogenomics and Personalized Medicine is an international, peer-reviewed, open access journal characterizing the influence of genotype on pharmacology leading to the development of personalized treatment programs and individualized drug selection for improved safety, efficacy and sustainability. This journal is indexed

\section{Dovepress}

on the American Chemical Society's Chemical Abstracts Service (CAS). The manuscript management system is completely online and includes a very quick and fair peer-review system, which is all easy to use. Visit http://www.dovepress.com/testimonials.php to read real quotes from published authors. 